\title{
ARTICLE
}

Received 9 Apr 2010 | Accepted 5 Aug 2010 | Published 7 Sep 2010

\section{Rapid calcium-dependent activation of Aurora-A kinase}

\author{
Olga V. Plotnikova', Elena N. Pugacheva², Roland L. Dunbrack \& Erica A. Golemis ${ }^{1}$
}

Oncogenic hyperactivation of the mitotic kinase Aurora-A (AurA) in cancer is associated with genomic instability. Increasing evidence indicates that AurA also regulates critical processes in normal interphase cells, but the source of such activity has been obscure. We report here that multiple stimuli causing release of $\mathrm{Ca}^{2+}$ from intracellular endoplasmic reticulum stores rapidly and transiently activate AurA, without requirement for second messengers. This activation is mediated by direct $\mathrm{Ca}^{2+}$-dependent calmodulin ( $\mathrm{CaM}$ ) binding to multiple motifs on AurA. On the basis of structure-function analysis and molecular modelling, we map two primary regions of CaM-AurA interaction to unfolded sequences in the Aur A N- and C-termini. This unexpected mechanism for AurA activation provides a new context for evaluating the function of AurA and its inhibitors in normal and cancerous cells. 
T he Aurora-A (AurA) kinase is a member of the evolutionarily conserved Ipl family of kinases (reviewed in Marumoto et al. ${ }^{1}$ ). AurA is most abundant at the centrosome in $\mathrm{G} 2$ to $\mathrm{M}$ phase $\mathrm{e}^{2}$, and in studies performed in mammals and model organisms including Drosophila, AurA has been shown to be activated at mitotic entry, performing critical functions in regulating entry into and passage through mitosis ${ }^{1,3}$. Activation of AurA at mitosis is supported by a complex set of interactions between the protein and a number of partners, including $\mathrm{Ajuba}^{4}, \mathrm{Pak}^{5}, \mathrm{Bora}^{6}$, TPX2 $2^{7}, \mathrm{PPI}^{8}$ and NEDD9 ${ }^{9}$. These interactions typically occur at the centrosome, with the partner proteins supporting AurA localization and stability, and inducing allosteric changes that contribute to AurA activation. Because of the complex nature of the interactions, the exact mechanism timing the abrupt AurA activation at the mitotic boundary has not been well resolved.

In the past several years, AurA has attracted increasing attention because it has been found to be overexpressed and hyperactivated in a high percentage of tumours arising in breast, colon, ovary and other tissues ${ }^{10-14}$. Abnormally high AurA activity is oncogenic in various cell line models and is associated with defective cytokinesis and aneuploidy ${ }^{15-18}$. AurA is now being actively exploited as a target for development of new anti-cancer agents (reviewed in Andrews ${ }^{19}$ ) on the basis of this known role as a mitotic regulator. Interestingly, the overexpressed AurA associated with cancer cells has a number of activities that are not specific to function in a mitotic compartment. For example, AurA directly phosphorylates and regulates the activity of the RalA GTPase, an EGFR/Ras effector important in many cancers ${ }^{20}$, with this activity observed in interphase cells. AurA is not typically mutationally activated in cancer, making the mechanism of its activation in interphase cells somewhat elusive.

Intriguingly, a number of studies have emerged in recent years to challenge the idea that AurA is solely a mitotic kinase even in normal cells. Serum induces AurA activation at the basal body of the cell cilium in non-cycling G0/G1-phase mammalian cells, causing AurA-dependent ciliary resorption ${ }^{21}$, and hence indirectly impacting the functionality of the cilia-dependent and cancer-relevant Hedgehog signalling cascades ${ }^{22}$. AurA also has been reported to regulate microtubule dynamics in interphase cells $^{23}$, and has been shown to be abundantly expressed in some human adult non-cycling tissues such as kidney ${ }^{24}$. All these studies strongly imply a non-mitotic activity for AurA, and indeed, a distant ortholog of AurA in the green algae Chlamydomonas regulates both resorption and excision of the flagella (a structure analogous to the mammalian cilium) in response to altered ionic conditions or cues for mating 25 , again suggesting important non-mitotic roles for AurA, although across a great evolutionary distance. However, to date, little effort has been made to investigate the non-mitotic activation of AurA in either normal cells or tumours, and mechanistic insight into factors governing such activation is essentially absent.

Our previous work demonstrating that AurA activation occurs before ciliary resorption in interphase cells ${ }^{21}$ was based on studies in Chlamydomonas, which implicated an AurA ortholog, CALK, as important for resorption of flagella in that organism ${ }^{25}$. Stimuli that lead to loss of flagella in Chlamydomonas include mating response to pheromone and transient ionic shock ${ }^{25}$. Recent studies in Chlamydomonas have also suggested increasing intraflagellar calcium concentrations during the mating response ${ }^{26}$, shortly before the activation of the CALK kinase. Independently, a study of the Chlamydomonas flagellar excision process emphasized the importance of rapid spatiotemporal patterning of calcium ion distribution as a critical mediating signal ${ }^{27}$. Interestingly, a recent study of oocyte maturation in Xenopus indicated that inhibition of $\mathrm{Ca}^{2+}$ signalling led to eventual failure to accumulate and activate $\operatorname{AurA}^{28}$, although no direct connection was investigated. Cumulatively, these studies led us to hypothesize that AurA might be a direct target of cellular calcium signalling in mammals.

Our work presented here demonstrates that elevated cytoplasmic calcium signals are transmitted rapidly and transiently through calmodulin (CaM) to activate AurA. This activation involves direct CaM binding to two sites in the unstructured regions of AurA, and induced by multiple inducers of calcium release from intracellular stores in the endoplasmic reticulum (ER). These results provide the first clear mechanism for AurA activation in non-mitotic cells and potential insight into the timing of activation of AurA at mitosis.

\section{Results}

ER release of $\mathrm{Ca}^{2+}$ rapidly and transiently activates AurA. Rapid and transient $\mathrm{Ca}^{2+}$ channel activity is stimulated by treatment of kidney cells with the peptide hormone and anti-diuretic arginine vasopressin (AVP) ${ }^{29,30}$. AVP signals through the AVPR1 receptor to initiate InsP3 and other second-messenger cascades that initiate $\mathrm{Ca}^{2+}$ release from the ER. The AVPR1 receptor is well expressed in the renal cell lines HK-2 and HEK293 (Fig. 1a). To assess whether activation of AurA occurred in this physiological context, we treated the HK-2 proximal tubule-derived human kidney cell line with AVP, and used immunofluorescence with antibody directed to $\mathrm{T}^{288}$ (auto-phosphorylated)-AurA to measure AurA activation (Fig. 1b). Strikingly, T ${ }^{288}$-phospho AurA at centrosomes increased threefold within $5 \mathrm{~s}$ of AVP treatment, returning to basal levels after $\sim 2 \mathrm{~min}$ (Fig. 1c), whereas total AurA levels were not affected (Fig. 1d). AurA activation might be mediated by $\mathrm{Ca}^{2+}$ influx from outside the cell, or by $\mathrm{Ca}^{2+}$ released from internal ER stores. Using AurAtransfected HEK293 cells, we found an equivalent degree of AurA activation after AVP treatment of cells cultured in medium with and without $\mathrm{Ca}^{2+}$ (Fig. 1e). To confirm that activation was not specific to the AVP-initiating stimulus, we also treated these cells grown in $\mathrm{Ca}^{2+}$-free medium with histamine, an InsP3 generating agonist that triggers activation of ER-associated calcium ion channels such as the IP3 receptor ${ }^{31}$. Again $\mathrm{T}^{288}$-AurA appeared rapidly after stimulation (Fig. 1f).

The fact that rapid AurA activation occurred after stimulation of cells grown without extracellular $\mathrm{Ca}^{2+}$ implied that the elevated cytoplasmic $\mathrm{Ca}^{2+}$ levels resulting from ER release might regulate AurA activation without intrinsic requirement for second messengers transmitting signals from membrane-localized receptors. To further assess this mechanism, we treated AurA-transfected HEK293 cells with the $\mathrm{Ca}^{2+}$ uptake inhibitor thapsigargin, which blocks the recycling of cytoplasmic $\mathrm{Ca}^{2+}$ into ER stores ${ }^{32}$, thus increasing cytoplasmic $\mathrm{Ca}^{2+}$ levels without the involvement of upstream activators. Thapsigargin treatment caused a rapid $(<1 \mathrm{~min})$ increase in levels of activated $\mathrm{T}^{288}$-AurA, and increased the in vitro kinase activity of immunoprecipitated AurA against defined substrates (Fig. 2a,b).

As another test, the BAPTA-AM Ca ${ }^{2+}$ ion chelator blocks $\mathrm{Ca}^{2+}$ mediated signalling pathway activation ${ }^{33}$; BAPTA-AM used alone or at low concentration in combination with EGTA completely blocked thapsigargin-induced $\mathrm{T}^{288}$-AurA auto-phosphorylation (Fig. 2c). In the absence of BAPTA-AM, EGTA (which chelates only extracellular $\mathrm{Ca}^{2+}$ ) had no effect either used alone or in conjunction with thapsigargin (Fig. 2d). In addition, treatment with the $\mathrm{Ca}^{2+}$-selective ionophore, ionomycin, which directly triggers $\mathrm{Ca}^{2+}$ release, similarly induced transient activation of AurA with similar kinetics (Fig. 2e). Further, a small molecule inhibitor of AurA, PHA680632 $2^{34}$, blocked thapsigargin- or ionomycin-induced AurA auto-phosphorylation (Fig. 2f).

The AurA partner protein, NEDD9, has been shown to be important for AurA activation in mitosis and in ciliary resorption $^{9,21}$, and been shown to be rapidly tyrosine phosphorylated (associated with more efficient function as an activator for some NEDD9 partner proteins) after $\mathrm{Ca}^{2+}$ release in osteoclasts ${ }^{35,36}$. 


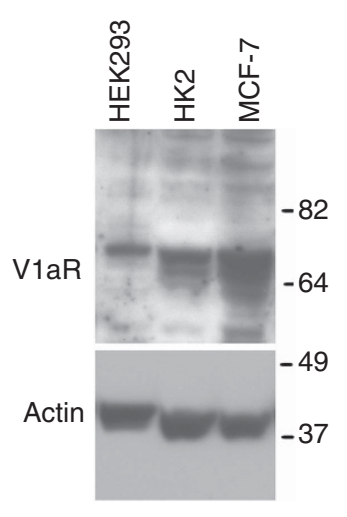

b

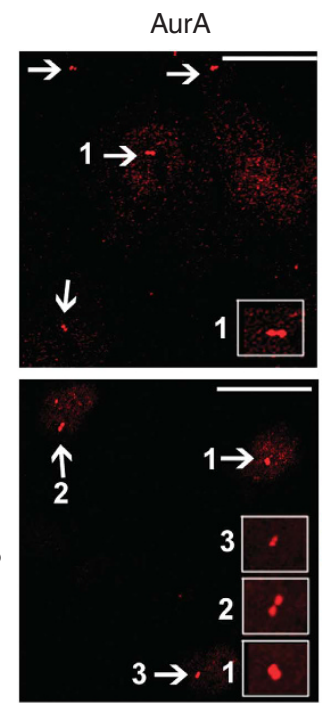

phAurA

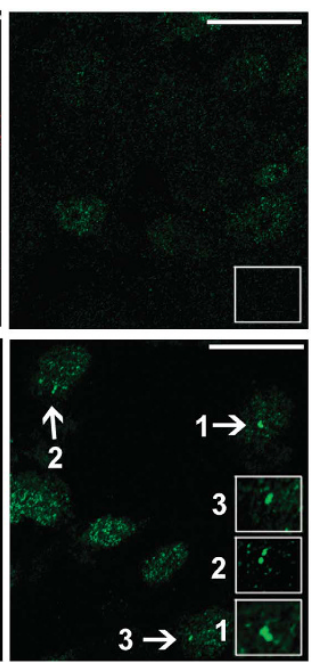

DAPI
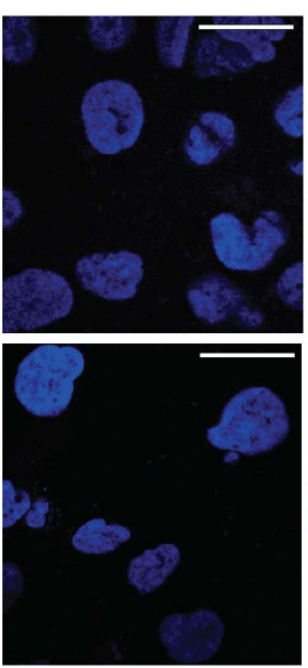

Merge

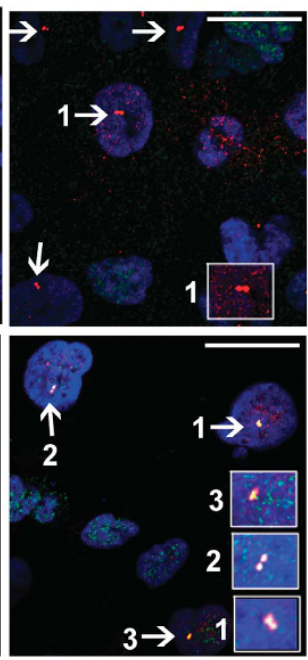

C

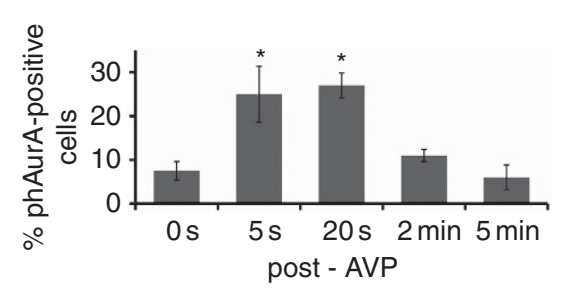

e
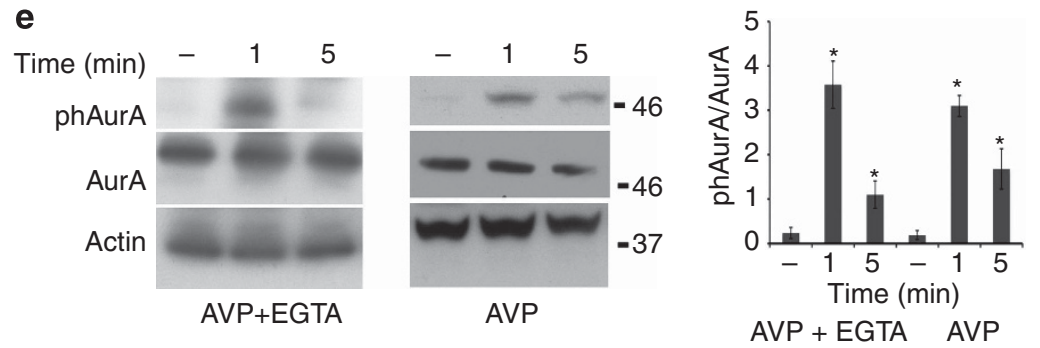

AVP + EGTA AVP
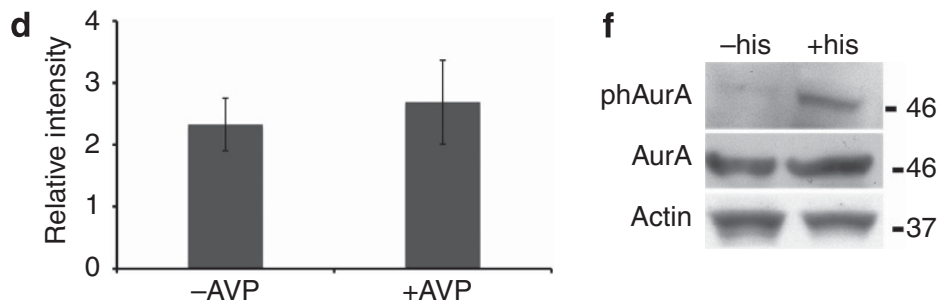

Figure 1 | Calcium release rapidly induces AurA auto-phosphorylation. (a) Western blot assay confirming expression of the AVPR1 receptor in cell lines used in this study. MCF7 were used as a positive control for AVPR1 expression, based on North et al. ${ }^{60}$. (b) Immunofluorescence of HK-2 cells $30 \mathrm{~s}$ after stimulation with $100 \mathrm{nM}$ arginine vasopressin ( + AVP) versus control untreated cells ( - AVP). Cells were visualized with antibodies to AurA (red) and $T^{288}$-phospho-AurA (phAurA, green) and DAPI (blue), as indicated; scale bar, $20 \mu \mathrm{m}$. Insets show magnification of indicated centrosomes. (c) Analysis of data from $\mathbf{b}$ quantifies phAurA-positive cells at times after stimulation. (d) Analysis of data from $\mathbf{b}$ quantifies relative intensity of AurA in cells within $20 \mathrm{~s}$ after stimulation with AVP. (e) Western blot analysis of AVP-treated HEK293 cells overexpressing AurA in the presence or absence of extracellular Ca ${ }^{2+}$ chelator ( 5 mM EGTA) (f) Western blot analysis of phAurA in HEK293 cells transfected with an AurA-expressing plasmid 1-2 min after stimulation with $10 \mu \mathrm{M}$ histamine. For each SDS-PAGE, $\beta$-actin is used as a loading control and molecular weight is indicated in $\mathrm{kDa}$. For $\mathbf{c}$ and $\mathbf{d}$, an average of 150 cells were counted. (c, d, e) $N=3$ with error bars indicating the standard error. ${ }^{\star} P<0.05$.

However, in cells transfected with small interfering RNA-depleting NEDD9, thapsigargin induced comparable AurA phosphorylation to that in control transfected cells (Fig. 2g). These data indicated that calcium-activated AurA phosphorylation is independent from NEDD9 activity, causing us to investigate other activation mechanisms.

$\mathrm{Ca}^{2+}$ induces $\mathrm{CaM}$ to bind and activate AurA. Increased cytoplasmic $\mathrm{Ca}^{2+}$ might induce AurA activation by direct binding or by inducing conformational changes in AurA based on AurA binding to a $\mathrm{Ca}^{2+}$ effector such as CaM. In an in vitro kinase assay, titration of $\mathrm{Ca}^{2+}$ into the reaction did not affect AurA auto-phosphorylation or activity towards substrates such as histone H3 (Fig. 3a). However, addition of $\mathrm{Ca}^{2+}$ with CaM strongly induced AurA auto-phosphorylation, as well as AurA phosphorylation of multiple canonical substrates including histone H3, MBP and NEDD9
(Fig. 3a-c). In addition, the AurA inhibitor PHA680632 also blocked CaM-induced phosphorylation (Fig. 3c).

These results predicted direct AurA-CaM binding. This binding was confirmed both in vitro and in vivo (Fig. 3d, e), and was positively regulated by $\mathrm{Ca}^{2+}$ (Fig. 3e). Treatment with PHA680632 had no effect on AurA-CaM binding (Fig. 3f), as would be expected based on defined PHA680632 interactions with a unique site in the hinge region of the AurA catalytic domain ${ }^{37}$. CaM pulldown was specific to AurA, as a much weaker pulldown was seen with the related Ipl kinase AurB and no pulldown was seen with an unrelated kinase, Src (Fig. 3g). Further, thapsigargin-induced activation of endogenous AurA in HK-2 cells and of transfected AurA in HEK293 cells was completely blocked by the CaM inhibitor calmidazolium (Fig. 3h, i). Using mass spectrometry, we determined that addition of CaM to AurA resulted in the appearance of a substantial $S^{51}$ or $S^{53}$ phosphorylated AurA species (QRVLCP-S(51)-NSSQR), as well as 
a

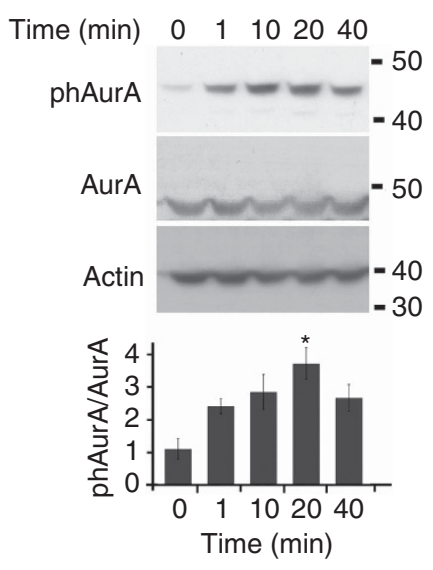

d

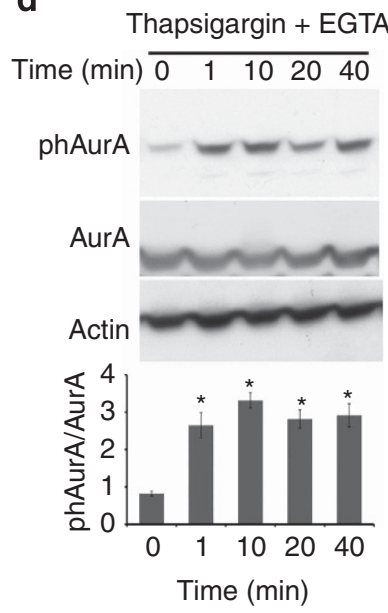

b
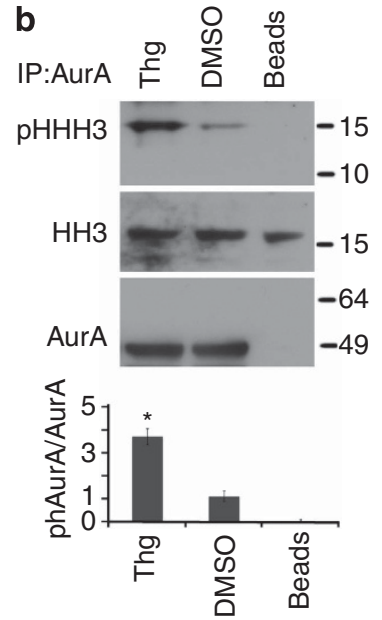

c

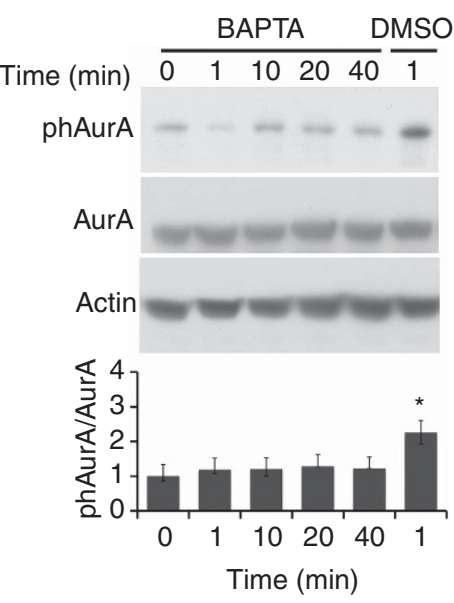

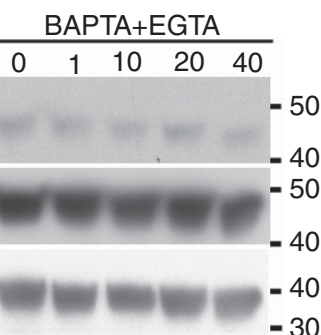

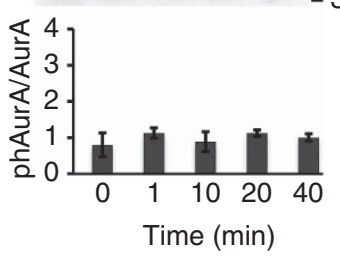

\section{年} tions 30

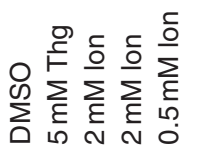

Time (min) $0 \begin{array}{llll}0 & 5 & 5 & 2\end{array}$
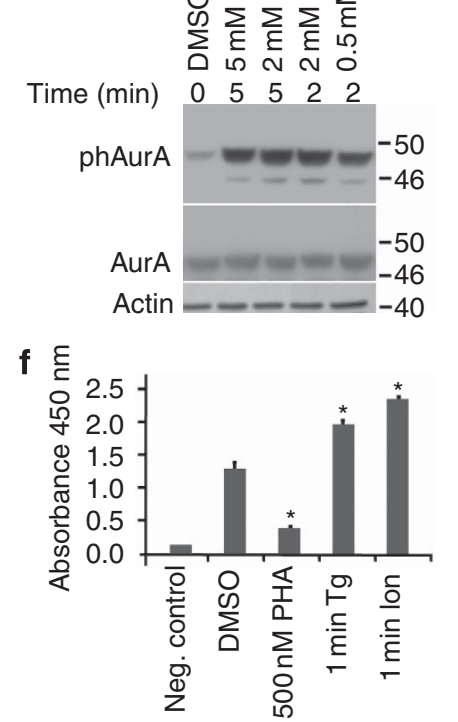

e g

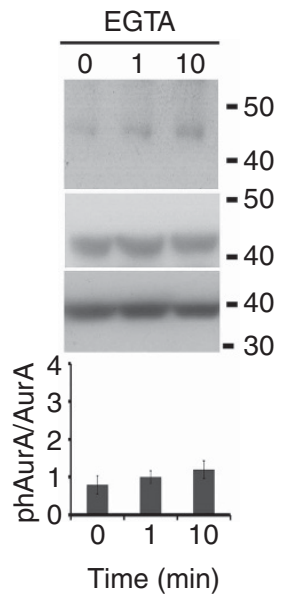

Figure $\mathbf{2}$ | Intracellular calcium release is sufficient for AurA activation. (a) HEK293 cells overexpressing AurA were incubated with $5 \mu M$ thapsigargin for the indicated periods of time, and then cell lysates were analysed by western blot. Graph shown below indicates ratio of phAurA to total AurA. (b) AurA immunoprecipitation and in vitro kinase assay, after thapsigargin treatment. HEK293 cells overexpressing AurA were treated with $5 \mu \mathrm{M}$ thapsigargin or control dimethylsulpfoxide (DMSO) for $5 \mathrm{~min}$, Aur A was immunoprecipitated and incubated with histone $\mathrm{H} 3$ substrate in an in vitro kinase assay. phHH3, antibody to phosphorylated histone H3. ${ }^{*} P<0.05$. (c) Left, an experiment similar to that shown in $\mathbf{a}$, except that it is performed in the presence of $50 \mu \mathrm{M}$ BAPTA-AM or control DMSO. Right, HEK293 cells overexpressing AurA were incubated with $20 \mu \mathrm{M}$ BAPTA-AM (30 min) plus 7.5 mM EGTA ( $2 \mathrm{~min}$ ) before the addition of $5 \mu \mathrm{M}$ thapsigargin for the indicated periods of time, with analysis as for $\mathbf{a}$. (d) An experiment similar to that shown in $\mathbf{a}$, except that it is performed in the presence of $5 \mathrm{mM} \mathrm{EGTA}$ alone for the indicated periods of time (right panel) or in the presence of $5 \mathrm{mM}$ EGTA ( 2 min) before the addition of $5 \mu \mathrm{M}$ thapsigargin. (e) HEK293 cells overexpressing AurA were incubated with $5 \mu \mathrm{M}$ thapsigargin and indicated concentration of ionomycin for the indicated periods of time, and then cell lysates were analysed by western blot using indicated antibodies. (f) HK2 cells were treated with DMSO, $5 \mu \mathrm{M}$ thapsigargin ( $\mathrm{Tg}$ ) or $0.5 \mu \mathrm{M}$ ionomycin (Ion), and then $\mathrm{T}^{288}$-phAurA levels were analysed by enzyme-linked immunosorbent assay (ELISA), based on $\mathrm{A}_{450 \mathrm{~nm}}$. To confirm the specificity of ELISA signal, a parallel group of HK2 cells were pre-treated with the AurA inhibitor PHA680632 (PHA) at $500 \mathrm{nM}$ concentration. ${ }^{\star} P<0.05$. Lysis buffer was used as a negative control. (g) AurA-overexpressing HEK293 cells were transfected with siRNAdepleting NEDD9 (siNEDD9) or scrambled control siRNA (scr), and then were incubated with 5- $\mu \mathrm{m}$ thapsigargin and processed as in $\mathbf{a}$. For this and subsequent SDS-PAGE analyses, each experiment was performed three times independently, with error bars indicating the standard error. ${ }^{\star} P<0.05$.

additional phosphorylation events on $\mathrm{S}^{66}$ or $\mathrm{S}^{67}$, and $\mathrm{S}^{98}$. Interestingly, phosphorylation of $S^{53}$ of the Xenopus AurA ortholog (analogous to $S^{51}$ in human AurA) has been previously shown to be a product of mitotic auto-phosphorylation, and to stabilize AurA from proteasomal degradation at mitotic exit ${ }^{38,39}$.

Definition of CaM-binding sites on AurA. CaM binding is typically modulated by a combination of defined amino acid motifs and a disordered secondary structure with the potential to form an $\alpha$-helix ${ }^{40}$. We used four programs for predicting intrinsically disordered regions in proteins (IUPRED, MetaPRDOS, PONDR_FIT and VL3) to analyse AurA structure (Fig. 4a). This analysis showed that consensus regions encompassed within approximately residues 1-127 and 385-403 were predicted to be disordered. A crystal structure of residues 100-403 of AurA has been determined ${ }^{37}$ (Protein Data Bank entry 2J4Z), and only residues 126-389 are seen in the 
a

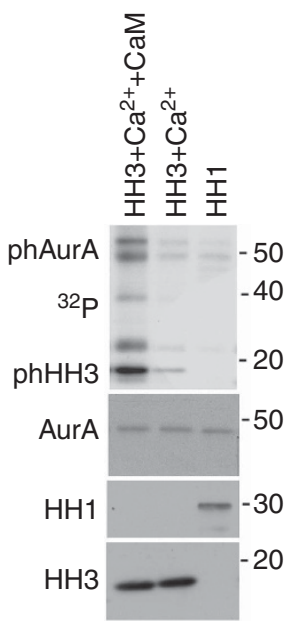

b

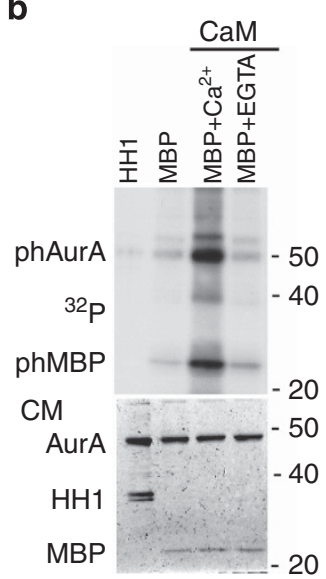

c

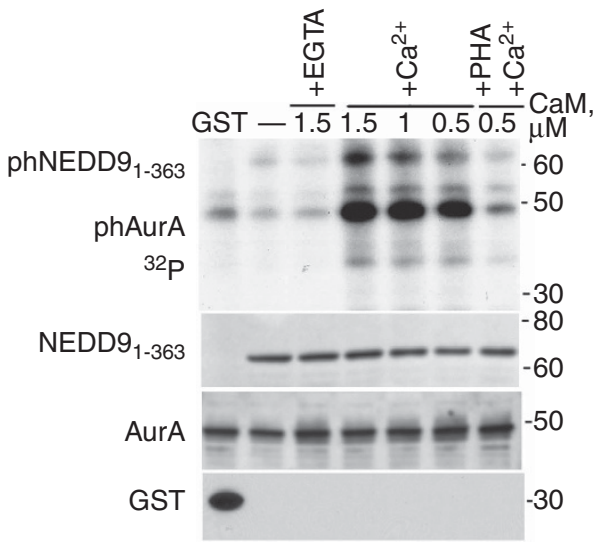

d

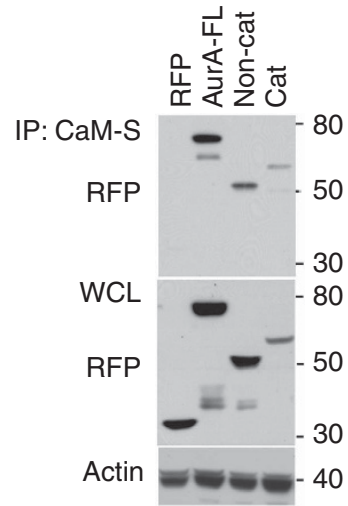

e

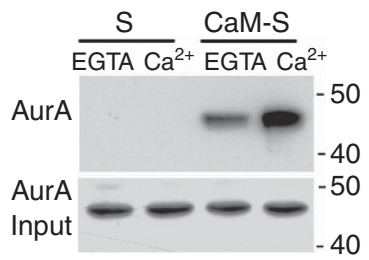

f

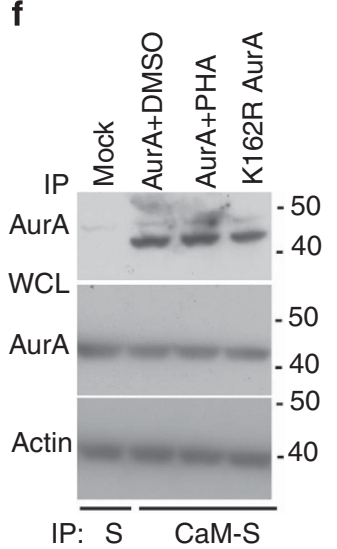

g

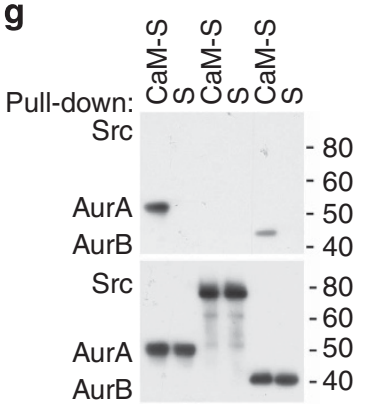

h
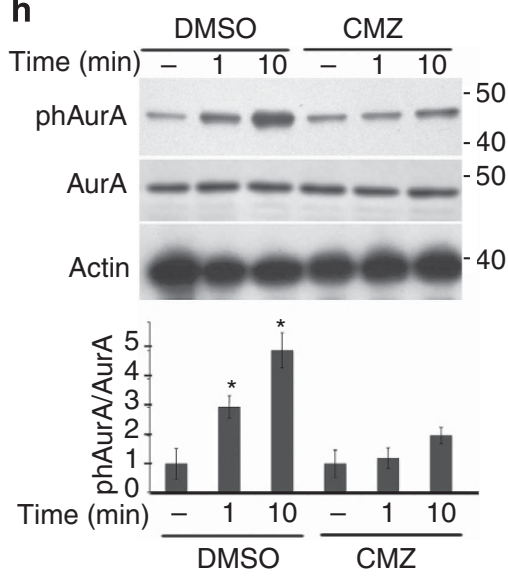

Figure 3 | CaM binds and activates AurA. (a) In vitro kinase assay using recombinant AurA, with the addition of $\mathrm{Ca}^{2+}$ or $\mathrm{CaM}$ as indicated, with histone $\mathrm{H} 3(\mathrm{HH} 3)$-positive and histone $\mathrm{H} 1(\mathrm{HH} 1)$-negative control. ${ }^{32}$ P- $\gamma$-ATP signal at $~ 55 \mathrm{kDa}$ indicates AurA auto-phosphorylation. Similar activation of AurA was obtained with concentrations of $\mathrm{Ca}^{2+}$ as low as $0.5 \mu \mathrm{M}$. (b) In vitro kinase assay using recombinant AurA, with the addition of $\mathrm{CaM}$ and $\mathrm{Ca}{ }^{2+}$ as indicated, with MBP used as Aur A substrate and histone $\mathrm{H} 1$ as negative control. (c) In vitro kinase assay shows that CaM activates Aur A. Top, ${ }^{32} \mathrm{P}-\gamma$-ATP signal at $\sim 55 \mathrm{kDa}$ indicates AurA auto-phosphorylation, and signal at $\sim 55 \mathrm{kDa}$ indicates phosphorylation of NEDD9 ${ }_{1-363}$ substrate. Bottom, representation of all indicated proteins in the reaction mixture. The AurA inhibitor PHA680632 (PHA) was used at 1 $\mu$ M. (d) CaM-Sepharose (CaM-S) was used for immunoprecipitation (IP) from whole cell lysates (WCL) transfected with plasmids expressing red fluorescent protein (RFP), AurA-RFP and RFP-fused N-terminal (non-cat) and C-terminal (cat, kinase domain) of AurA, followed by western blotting. (e) AurA pulldown with CaM-Sepharose is $\mathrm{Ca}^{2+}$ dependent. Recombinant AurA plus $1 \mathrm{mM} \mathrm{CaCl}\left(\mathrm{Ca}^{2+}\right)$ or $1 \mathrm{mM} \mathrm{EGTA}$ was incubated with CaM-Sepharose (CaM-S) or control Sepharose (S). (f). Experiment is carried out as in e, except that AurA is incubated with dimethylsulpfoxide (DMSO) or the AurA kinase inhibitor, PHA-680632, or a kinase-dead mutant of AurA (K162R) is used for pulldown. (g) Experiment is carried out as in e, performed in the presence of $1 \mathrm{mM} \mathrm{Ca}{ }^{2+}$. (h) Western blot of lysates from HEK293 cells overexpressing Aur A were preincubated for $1 \mathrm{~h}$ with $10 \mu \mathrm{M}$ of the CaM inhibitor calmidazolium (CMZ) or control DMSO, incubated with $5 \mu \mathrm{M}$ thapsigargin. Graph indicates ratio of phAurA to total AurA, quantified from analysis of three blots; error bars show standard error; ${ }^{\star} P<0.05$. (i) $\mathrm{HK} 2$ cells were preincubated for $1 \mathrm{~h}$ with $10 \mu \mathrm{M} \mathrm{CMZ}$ or DMSO, treated with 5 - $\mu \mathrm{m}$ thapsigargin for 1 min and then analysed by immunofluorescence with antibodies to AurA and T288-phospho-AurA. An average of 150 cells were counted in each of three experiments, with error bars indicating the standard error. ${ }^{\star} P=0.00054$.

density map, confirming the disorder prediction. These residues also coincide with the canonical $\mathrm{N}$ - and C-terminal kinase domains, for example, as defined by the PFAM database ${ }^{41}$. In addition, we used the secondary structure prediction program PSIPRED ${ }^{42,43}$ on a set of AurA orthologs from mammals and Xenopus (Fig. 4b), identifying two regions of helical propensity in the $\mathrm{N}$-terminal disordered region, consisting of residues 59-82 and 111-127 in the human sequence. The C-terminal disordered region is predicted to be coiled, and not an $\alpha$-helix or $\beta$-sheet. However, just before this region, residues 353-364 and 367-387 are $\alpha$-helices in the experimental structure.

Further refinement of this analysis allowed the mapping of a strong minimal interaction domain to residues $33-89$, and suggested weaker binding elements encompassed within residues 82-132, 350-368 and 368-403 (Fig. 4c,d). Suggestively, the minimum strong CaM-AurA-binding site encompassed the longest of the predicted $\alpha$-helices in the $\mathrm{N}$-terminal disordered region (residues 59-82), with the $\alpha$-helices adjacent to or containing the $\mathrm{Ca}^{2+}$ dependent auto-phosphorylation sites $\left(S^{51} / S^{53}\right.$ and $\left.S^{66} / S^{67}\right)$. Further, the $\alpha$-helix, the phosphorylation sites and the flanking sequences show striking conservation through mammals (Fig. 4b), implying an important function. The weaker binding of CaM to the other minimal binding elements may be due to unfolding of the protein in some constructs; for example, the predicted helical regions in residues 350-368 are encompassed in the catalytic domain, and would 
normally be folded. However, we cannot rule out local unfolding of this region and $\mathrm{CaM}$ binding in intact AurA, based on interactions of AurA with partner proteins.

\section{Discussion}

The work presented here reveals and mechanistically defines a novel role for AurA as a component of rapid calcium signalling responses. In interphase cells, we propose that transient stimuli such as AVP

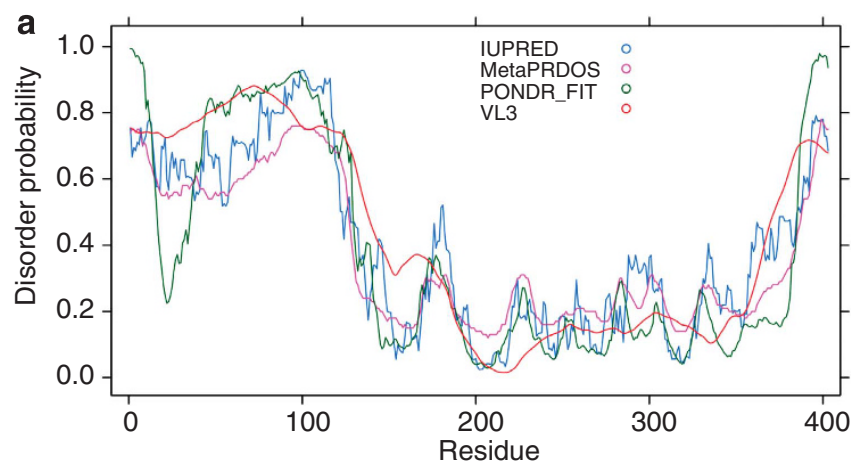

b
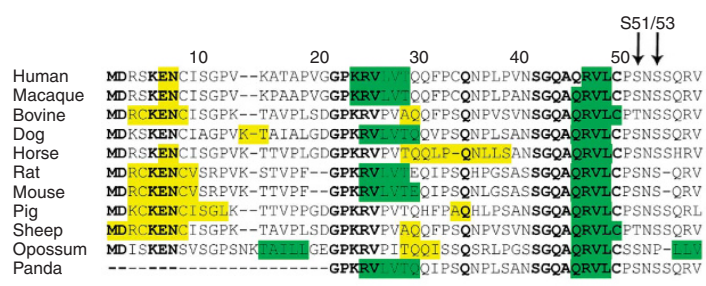

$\mathrm{S} 66 / 67$

S98
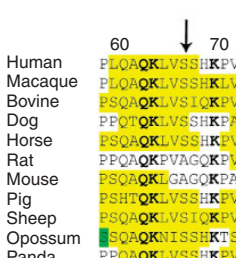

100

$10 \quad 120$
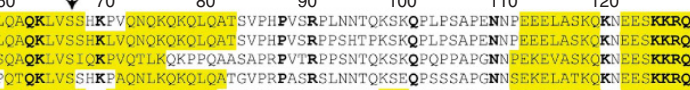

QKLVSSHKPVQNLKQKQSQATSVPHPVPRPLNNT QRSEQ Q LPSAPGNNSEKELASKEKNEESKKRQ

QAQKPVAGQKPVL----KQLPAASGPRPASR-LSNPQKSEQPQPAASGNNSEKEQTS I OKTEDSKKRQ

QRKLGAGQKPAP----KQLPAASVRR VSR-LNNPQKNEQP--AASGNDSEKEQASLOKTEDTKKRQ

QAQK VSIQK PVQTLKQKPPQAASAPRPVARPPSNTQKSKQPQPPAPGNNPEKEVASKQKNEESKKRQ

SQAQKNISSHKT SHAHQSOKLQQAPVIHPTSRPPSSTQNTEQSQP PVSGNIPGKGETPROKNEETKKRQ

Panda PPQAQK VSSHKPVQNLKQKQLQATSVPRPVSRPLNNTQKNEQASSSAPGNNSEKE LATKQKNEESKKRQ

C
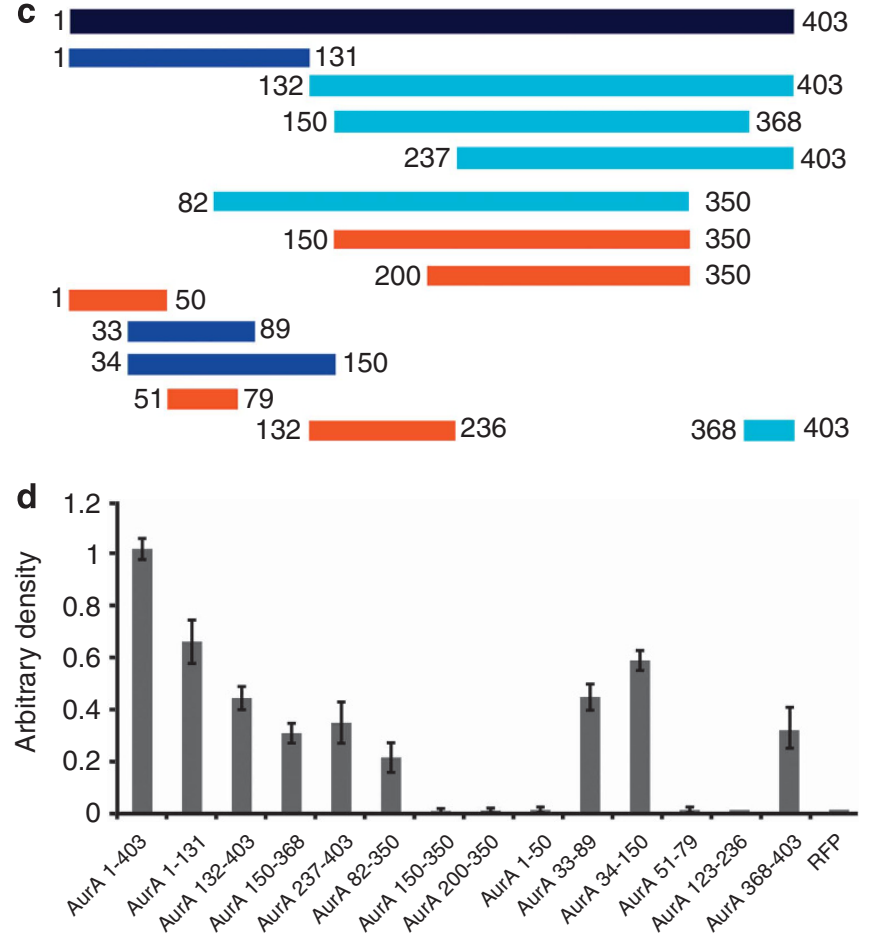

or histamine trigger $\mathrm{Ca}^{2+}$ release into the cytoplasm, inducing $\mathrm{CaM}$ binding and auto-activation of AurA. These activities involve different activation mechanisms than those we have previously reported for AurA in regulation of ciliary disassembly ${ }^{21}$, occurring with much faster kinetics (2-5 min versus $1-2 \mathrm{~h}$ ) and not influenced by depletion of NEDD9. Although our data do not exclude the possibility that second messengers or cilia-based signalling systems may contribute to the activation of AurA in calcium response, they argue against an essential requirement for such activation. Such transient activation of AurA in response to short-term increases in cytoplasmic calcium may offer a mechanism by which AurA is able to phosphorylate targets such as $\mathrm{RalA}^{20}$ and microtubules ${ }^{23}$ in non-mitotic cells. Interestingly, RalA is itself regulated by $\mathrm{Ca}^{2+}$ in a non-Ras-dependent activation pathway ${ }^{44}$, and RalA activity has been reported to be controlled by $\mathrm{CaM}$ binding ${ }^{45}$. Our data raise the possibility that parallel CaM-dependent activation of AurA may help control the degree and timing of RalA activation in cancer and normal cells. The activation of AurA by $\mathrm{Ca}^{2+}-\mathrm{CaM}$ may also have implications for AurA functions at cilia. The polycystic kidney disease (PKD)-associated genes PKD1 and PKD2 encode cilia-associated, heterodimerizing proteins that function as mechanosensors, internalizing $\mathrm{Ca}^{2+}$ in response to flow-based cues ${ }^{46}$. This localized release of $\mathrm{Ca}^{2+}$ may transiently activate AurA, given the proximal location of the AurA protein at the ciliary basal body, allowing AurA to phosphorylate substrates relevant to the mechanosensing process. For example, AurA exists in a stable complex with NEDD9, and NEDD9 (HEF1) has previously been reported to bind the Id $2^{47}$, a protein that is phosphorylated and influences cell differentiation in response to PKD1/PKD2-mediated signalling ${ }^{48}$.

These data also offer insight into control mechanisms operant in the cell cycle-regulated activation of AurA. Dynamic changes in calcium signalling have a key role in meiosis, have been implicated in action at spindle-associated microdomains in mitosis ${ }^{49}$ and may also regulate AurA. An attractive feature of $\mathrm{Ca}^{2+}$-dependent AurA regulation is that it offers a potential mechanism to help explain the rapid, timed activation of AurA at cell cycle transitions. At present, although multiple proteins have been demonstrated to bind and support AurA activation at the mitotic boundary, most of these also interact with AurA in G2, implying the existence of a triggering event at the actual transition point. The transient increase of cytoplasmic $\mathrm{Ca}^{2+}$ could provide a suitable mitotic trigger. Such $\mathrm{Ca}^{2+}$ and $\mathrm{CaM}$-dependent regulation of the centrosomal proteins centrin and CP110 has been shown to be critical for the action of these proteins in supporting cytokinesis. In this view, $\mathrm{Ca}^{2+}$ release and $\mathrm{CaM}$ binding may be a physiological trigger for AurA during cell cycle, causing conformational changes that are permissive for other AurAinteracting proteins to sustain its activity during long-term rather

\section{Figure 4 | Definition of multiple CaM-binding motifs on AurA.}

(a) Prediction of disordered regions in AurA, using programs IUPRED (blue), MEtaPRDOS (purple), PONDR_FIT (green) and VLE (red). Higher numerical values represent increased likelihood of disorder. (b) Sequence conservation in the AurA amino-terminus (1-127). Predicted $\beta$-sheets are shown in green and $\alpha$-helices in yellow. Arrows indicates $\mathrm{Ca}_{2}{ }^{+}$-dependent AurA auto-phosphorylation sites. Bold represents highly conserved amino acids. (c) Schematic representation of AurA variants used for $\mathrm{CaM}$-binding studies. Intensity of binding is indicated by blue shading, with dark blue indicating the strongest binding, based on data in c. Red indicates no binding. (d) Quantitation of binding of AurA domains with CaM. Graph indicates ratio of coimmunoprecipitated AurA to total AurA, quantified from analysis of blots from three independent experiments in which CaM-Sepharose was used for immunoprecipitation from whole cell lysates expressing red fluorescent protein (RFP), AurA-RFP and RFP-fused domains of AurA, followed by western blotting with antibodies to RFP. Bars indicate standard error. 
than transient responses. Intriguingly, calcium signalling differs significantly in cancerous ${ }^{50}$ versus normal cells, promoting increased cell proliferation through the abnormal activation of numerous calcium-responsive signalling pathways. Hence, our work also raises the possibility that the enhanced AurA activity often found in tumours may, in part, arise from an abnormal calcium signalling environment. These points merit further investigation.

\section{Methods}

Plasmids and cell culture. FLAG- and glutathione S-transferase (GST)fused HEF1 were expressed from the vectors pCatch-FLAG ${ }^{51}$ and $p G E X-2 T^{52}$, respectively, as previously described. AurA and derivatives were expressed from pCMV-SPORT6-C6 (OpenBiosystems) and pcDNA3.1-mRFP vectors. A PCR product of mRFP was ligated into pcDNA3.1(+) (Invitrogen) to create pcDNA3.1mRFP. pcDNA3.1-mRFP was used as a negative control. Catalytically inactive AurA (K162R) was prepared using a site-directed mutagenesis kit (Stratagene). HEK293 cells were maintained in Dulbecco's modified Eagle's medium with 10\% fetal bovine serum plus penicillin/streptomycin. The immortalized human kidney proximal tubular cell line (HK-2, ATCC, cat. no. CRL-219) was grown to subconuence in keratinocyte media (Invitrogen). We transiently transfected HEK293 cells with expression constructs for HEF1 and AurA using Lipofectamine and Plus reagent (Invitrogen) according to the manufacturer's instructions.

Immunofluorescence. Cells growing on coverslips were fixed with $4 \%$ paraformal dehyde (10 min) and cold methanol (5 min), permeabilized with $1 \%$ Triton X-100 in phosphate-buffered saline (PBS), blocked in $1 \times$ PBS with $3 \%$ bovine serum albumin and incubated with antibodies using standard protocols. Primary antibodies included mouse anti-AurA (BD Biosciences; 1:200) and rabbit polyclonal antiphospho-AurA/T288 (Cell Signaling; 1:100). Secondary antibodies labelled with Alexa-488, Alexa-568 and DAPI (4,6-diamidino-2-phenylindole) to stain DNA, were from Molecular Probes/Invitrogen (1:2000). Confocal microscopy was performed using a Nikon $\mathrm{C} 1$ spectral confocal microscope (Nikon).

Western blotting and immunoprecipitation. Recombinant Hexa-histidine AurA was produced in a baculovirus expression system. For western blotting and immunoprecipitation, mammalian cells were disrupted in CelLytic M lysis buffer (Sigma) supplemented with a protease and phosphates inhibitor cocktails (Roche). Wholecell lysates were used either directly for SDS polyacrylamide gel electrophoresis (SDS-PAGE) or for immunoprecipitation. Immunoprecipitation samples were incubated overnight with antibody at $4^{\circ} \mathrm{C}$, and subsequently incubated for $2 \mathrm{~h}$ with protein $\mathrm{A} / \mathrm{G}$-sepharose (Pierce), washed and resolved by SDS-PAGE.

Western blotting was done using standard procedures, and developed by chemiluminescence using the West-Pico system (Pierce). Primary antibodies included mouse anti-NEDD9 mAb (clone 2G99; 1:1000), anti-AurA (BD Biosciences; 1:3000), anti-phospho-AurA-T ${ }^{288}$ and anti-GST mAb (Cell Signaling; $1: 2000)$, anti-V1aR (Santa Cruz Biotechnology; $1: 600)$ and anti- $\beta$-actin mAb (AC15, Sigma;1:20,000). Polyclonal anti-AurA agarose-immobilized conjugate (Bethyl) were used for immunoprecipitations. Secondary anti-mouse and antirabbit horseradish peroxidase-conjugated antibodies (GE Healthcare) were used at a dilution of 1:10,000 for visualization of western blots. Image analysis was done using NIH ImageJ-Image Processing and Analysis software (National Institutes of Health), with signal intensity normalized to $\beta$-actin or total AurA level.

Structural analysis. Four programs that predict intrinsically disordered regions were used via their webservers: IUPRED ${ }^{53}$, MetaPRDOS ${ }^{54}$, PONDR_FIT ${ }^{55}$ and $\mathrm{VL}^{56}{ }^{56}$. PSI-BLAST ${ }^{57}$ was used to search the Uniprot database ${ }^{58}$ to find orthologs of AurA and to identify crystal structures of AurA in the Protein Data Bank. A selection of these sequences was retrieved and a multiple sequence alignment was performed with Clustal $\mathrm{W}^{59}$. The program PSIPRED ${ }^{43}$ was used to predict secondary structure for these sequences based on single sequence predictions, rather than multiple sequence alignments from PSI-BLAST.

CaM association studies. Cell lysates in lysis buffer (PBS with $1 \%$ Triton X-100) or purified GST fusion protein diluted in binding buffer $(50 \mathrm{mM}$ Tris- $\mathrm{HCl}(\mathrm{pH} 7.6)$, $120 \mathrm{mM} \mathrm{NaCl}, 1 \%$ Brij) were incubated with CaM-Sepharose 4B (GE Healthcare) or control Sepharose for $1-2 \mathrm{~h}$ at $4{ }^{\circ} \mathrm{C}$, as indicated in the figure legends. In defined in vitro experiments, AurA was purified from baculovirus, as previously described $^{21}$, whereas recombinant proteins AurB or Src were obtained from Millipore. After washing, beads were boiled in SDS sample buffer and separated by SDS-PAGE followed by western blotting.

Phosphorylation assays. MBP, histone $\mathrm{H} 3$ (Upstate) and histone H1 (Upstate) were used as positive and negative controls for AurA phosphorylation using standard methods. Parallel aliquots without $\gamma^{-32} \mathrm{P}(\mathrm{ATP})$ were processed for SDS-PAGE/ Coomassie staining (Invitrogen). To assess CaM-dependent AurA activation, in vitro kinase assay was performed using AurA purified from baculovirus or according the protocol described above, in the presence of $1 \mu \mathrm{M} \mathrm{CaM}$ (Calbiochem) and $1 \mathrm{mM} \mathrm{Ca}^{2+}$. For kinase assay without $\mathrm{Ca}^{2+}, 1 \mathrm{mM}$ EGTA was substituted for
$1 \mathrm{mM} \mathrm{Ca}^{2+}$ in the kinase buffer. Endogenous phospho- ${ }^{288}$-AurA protein in HK2 cells was detected by PathScan Phospho-AurA Sandwich ELISA kit (Cell Signaling) according to the manufacturer's instructions.

Mass spectrometry. After an in vitro kinase reaction with AurA produced from baculovirus in the presence or absence of CaM plus $1 \mathrm{mM} \mathrm{CaCl}_{2}$, gels were stained with Coomassie blue and phosphorylated AurA bands were excised and sequenced at the Taplin Biological Mass Spectrometry Facility at Harvard Medical School, by using microcapillary reverse-phase HPLC nanoelectrospray tandem mass spectrometry on a Finnigan LCQ DECA quadrupole ion trap mass spectrometer.

Statistical analysis. Statistical comparisons were made using a two-tailed Student's $t$-test. Experimental values were reported as the means \pm s.e. Differences in mean values were considered significant at $P<0.05$. All calculations of statistical significance were made using the GraphPad InStat software package (GraphPad).

\section{References}

1. Marumoto, T., Zhang, D. \& Saya, H. Aurora-A - a guardian of poles. Nat. Rev. Cancer 5, 42-50 (2005).

2. Fukuda, T., Mishina, Y., Walker, M. P. \& DiAugustine, R. P. Conditional transgenic system for mouse aurora a kinase: degradation by the ubiquitin proteasome pathway controls the level of the transgenic protein. Mol. Cell. Biol. 25, 5270-5281 (2005).

3. Pugacheva, E. N. \& Golemis, E. A. HEF1-aurora A interactions: points of dialog between the cell cycle and cell attachment signaling networks. Cell Cycle 5, 384-391 (2006).

4. Hirota, T. et al. Aurora-A and an interacting activator, the LIM protein Ajuba, are required for mitotic commitment in human cells. Cell 114, 585-598 (2003).

5. Zhao, Z. S., Lim, J. P., Ng, Y. W., Lim, L. \& Manser, E. The GIT-associated kinase PAK targets to the centrosome and regulates Aurora-A. Mol. Cell 20, 237-249 (2005).

6. Hutterer, A. et al. Mitotic activation of the kinase Aurora-A requires its binding partner Bora. Dev. Cell 11, 147-157 (2006).

7. Bayliss, R., Sardon, T., Vernos, I. \& Conti, E. Structural basis of Aurora-A activation by TPX2 at the mitotic spindle. Mol. Cell 12, 851-862 (2003).

8. Satinover, D. L., Leach, C. A., Stukenberg, P. T. \& Brautigan, D. L. Activation of Aurora-A kinase by protein phosphatase inhibitor-2, a bifunctional signaling protein. Proc. Natl Acad. Sci. USA 101, 8625-8630 (2004).

9. Pugacheva, E. N. \& Golemis, E. A. The focal adhesion scaffolding protein HEF1 regulates activation of the Aurora-A and Nek2 kinases at the centrosome. Nat. Cell. Biol. 7, 937-946 (2005).

10. Bischoff, J. R. et al. A homologue of Drosophila aurora kinase is oncogenic and amplified in human colorectal cancers. EMBO J. 17, 3052-3065 (1998).

11. Zhou, H. et al. Tumour amplified kinase STK15/BTAK induces centrosome amplification, aneuploidy and transformation. Nat. Genet. 20, 189-193 (1998).

12. Goepfert, T. M. et al. Centrosome amplification and overexpression of aurora A are early events in rat mammary carcinogenesis. Cancer Res. 62, 4115-4122 (2002).

13. Tanner, M. M. et al. Frequent amplification of chromosomal region 20q12-q13 in ovarian cancer. Clin. Cancer Res. 6, 1833-1839 (2000).

14. Tanaka, T. et al. Centrosomal kinase AIK1 is overexpressed in invasive ductal carcinoma of the breast. Cancer Res. 59, 2041-2044 (1999).

15. Meraldi, P., Honda, R. \& Nigg, E. A. Aurora-A overexpression reveals tetraploidization as a major route to centrosome amplification in p53-/- cells. EMBO J. 21, 483-492 (2002).

16. Anand, S., Penrhyn-Lowe, S. \& Venkitaraman, A. R. AURORA-A amplification overrides the mitotic spindle assembly checkpoint, inducing resistance to Taxol. Cancer Cell 3, 51-62 (2003).

17. Zhang, D. et al. Cre-loxP-controlled periodic Aurora-A overexpression induces mitotic abnormalities and hyperplasia in mammary glands of mouse models. Oncogene 23, 8720-8730 (2004).

18. Tatsuka, M. et al. Multinuclearity and increased ploidy caused by overexpression of the aurora- and Ipl1-like midbody-associated protein mitotic kinase in human cancer cells. Cancer Res. 58, 4811-4816 (1998).

19. Andrews, P. D. Aurora kinases: shining lights on the therapeutic horizon? Oncogene 24, 5005-5015 (2005).

20. Wu, J. C. et al. Identification of V23RalA-Ser194 as a critical mediator for Aurora-A-induced cellular motility and transformation by small pool expression screening. J. Biol. Chem. 280, 9013-9022 (2005).

21. Pugacheva, E. N., Jablonski, S. A., Hartman, T. R., Henske, E. P. \& Golemis, E.A. HEF1-dependent Aurora A activation induces disassembly of the primary cilium. Cell 129, 1351-1363 (2007).

22. Wong, S. Y. et al. Primary cilia can both mediate and suppress Hedgehog pathway-dependent tumorigenesis. Nat. Med. 15, 1055-1061 (2009).

23. Lorenzo, C., Liao, Q., Hardwicke, M. A. \& Ducommun, B. Pharmacological inhibition of aurora-A but not aurora-B impairs interphase microtubule dynamics. Cell Cycle 8, 1733-1737 (2009).

24. Kurahashi, T., Miyake, H., Hara, I. \& Fujisawa, M. Significance of Aurora-A expression in renal cell carcinoma. Urol. Oncol. 25, 128-133 (2007). 
25. Pan, J., Wang, Q. \& Snell, W. J. An aurora kinase is essential for flagellar disassembly in Chlamydomonas. Dev. Cell 6, 445-451 (2004).

26. Huang, K. et al. Function and dynamics of PKD2 in Chlamydomonas reinhardtii flagella. J. Cell Biol. 179, 501-514 (2007).

27. Wheeler, G. L., Joint, I. \& Brownlee, C. Rapid spatiotemporal patterning of cytosolic $\mathrm{Ca} 2+$ underlies flagellar excision in Chlamydomonas reinhardtii. Plant J. 53, 401-413 (2008).

28. Sun, L. et al. Ca2+ homeostasis regulates Xenopus oocyte maturation. Biol. Reprod. 78, 726-735 (2008).

29. Cai, Y. et al. Calcium dependence of polycystin-2 channel activity is modulated by phosphorylation at Ser812. J. Biol. Chem. 279, 19987-19995 (2004).

30. Koulen, P. et al. Polycystin-2 is an intracellular calcium release channel. Nat. Cell Biol. 4, 191-197 (2002).

31. Thibonnier, M., Bayer, A. L., Simonson, M. S. \& Kester, M. Multiple signaling pathways of V1-vascular vasopressin receptors of A7r5 cells. Endocrinology 129, 2845-2856 (1991).

32. Sagara, Y. \& Inesi, G. Inhibition of the sarcoplasmic reticulum Ca2+ transport ATPase by thapsigargin at subnanomolar concentrations. J. Biol. Chem. 266 13503-13506 (1991)

33. Anyatonwu, G. I., Estrada, M., Tian, X., Somlo, S. \& Ehrlich, B. E. Regulation of ryanodine receptor-dependent calcium signaling by polycystin-2. Proc. Nat Acad. Sci. USA 104, 6454-6459 (2007).

34. Soncini, C. et al. PHA-680632 a novel Aurora kinase inhibitor with potent antitumoral activity. Clin. Cancer Res. 12, 4080-4089 (2006).

35. Zhang, Z., Hernandez-Lagunas, L., Horne, W. C. \& Baron, R. Cytoskeletondependent tyrosine phosphorylation of the p130(Cas) family member HEF1 downstream of the $\mathrm{G}$ protein-coupled calcitonin receptor. Calcitonin induces the association of HEF1, paxillin, and focal adhesion kinase. J. Biol. Chem. 274 25093-25098 (1999).

36. Zhang, Z., Neff, L., Bothwell, A. L., Baron, R. \& Horne, W. C. Calcitonin induces dephosphorylation of Pyk2 and phosphorylation of focal adhesion kinase in osteoclasts. Bone 31, 359-365 (2002).

37. Fancelli, D. et al. 1,4,5,6-tetrahydropyrrolo[3,4-c]pyrazoles: identification of a potent Aurora kinase inhibitor with a favorable antitumor kinase inhibition profile. J. Med. Chem. 49, 7247-7251 (2006).

38. Littlepage, L. E. et al. Identification of phosphorylated residues that affect the activity of the mitotic kinase Aurora-A. Proc. Natl Acad. Sci. USA 99, 15440-15445 (2002).

39. Littlepage, L. E. \& Ruderman, J. V. Identification of a new APC/C recognition domain, the A box, which is required for the Cdh1-dependent destruction of the kinase Aurora-A during mitotic exit. Genes Dev. 16, 2274-2285 (2002)

40. Radivojac, P. et al. Calmodulin signaling: analysis and prediction of a disorderdependent molecular recognition. Proteins 63, 398-410 (2006).

41. Finn, R. D. et al. The Pfam protein families database. Nucleic Acids Res. 38 D211-D222.

42. McGuffin, L. J., Bryson, K. \& Jones, D. T. The PSIPRED protein structure prediction server. Bioinformatics 16, 404-405 (2000).

43. Jones, D. T. Protein secondary structure prediction based on position-specific scoring matrices. J. Mol. Biol. 292, 195-202 (1999).

44. Hofer, F., Berdeaux, R. \& Martin, G. S. Ras-independent activation of Ral by a $\mathrm{Ca}(2+)$-dependent pathway. Curr. Biol. 8, 839-842 (1998).

45. Clough, R. R., Sidhu, R. S. \& Bhullar, R. P. Calmodulin binds RalA and RalB and is required for the thrombin-induced activation of Ral in human platelets. J. Biol. Chem. 277, 28972-28980 (2002).

46. Harris, P. C. \& Torres, V. E. Polycystic kidney disease. Annu. Rev. Med. 60, 321-337 (2009).

47. Law, S. F. et al. Dimerization of the docking/adaptor protein HEF1 via a carboxy-terminal helix-loop-helix domain. Exp. Cell Res. 252, 224-235 (1999).
48. Li, X. et al. Polycystin-1 and polycystin-2 regulate the cell cycle through the helix-loop-helix inhibitor Id2. Nat. Cell Biol. 7, 1102-1112 (2005).

49. Parry, H., McDougall, A. \& Whitaker, M. Microdomains bounded by endoplasmic reticulum segregate cell cycle calcium transients in syncytial Drosophila embryos. J. Cell Biol. 171, 47-59 (2005).

50. Roderick, H. L. \& Cook, S. J. Ca2+ signalling checkpoints in cancer: remodelling $\mathrm{Ca} 2+$ for cancer cell proliferation and survival. Nat. Rev. Cancer 8, 361-375 (2008).

51. O’Neill, G. M. \& Golemis, E. A. Proteolysis of the docking protein HEF1 and implications for focal adhesion dynamics. Mol. Cell. Biol. 21, 5094-5108 (2001).

52. Law, S. F., Zhang, Y. -Z., Klein-Szanto, A. \& Golemis, E. A. Cell-cycle regulated processing of HEF1 to multiple protein forms differentially targeted to multiple compartments. Mol. Cell. Biol. 18, 3540-3551 (1998).

53. Dosztanyi, Z., Csizmok, V., Tompa, P. \& Simon, I. IUPred: web server for the prediction of intrinsically unstructured regions of proteins based on estimated energy content. Bioinformatics 21, 3433-3434 (2005).

54. Ishida, T. \& Kinoshita, K. Prediction of disordered regions in proteins based on the meta approach. Bioinformatics 24, 1344-1348 (2008).

55. Xue, B., Dunbrack, R. L., Williams, R. W., Dunker, A. K. \& Uversky, V.N. PONDR-FIT: a meta-predictor of intrinsically disordered amino acids. Biochim. Biophys. Acta 1804, 996-1010 (2010).

56. Peng, K. et al. Optimizing long intrinsic disorder predictors with protein evolutionary information. J. Bioinform. Comput. Biol. 3, 35-60 (2005).

57. Altschul, S. F. et al. Gapped BLAST and PSI-BLAST: a new generation of protein database search programs. Nucleic Acids Res. 25, 3389-3402 (1997).

58. Apweiler, R et al. The Universal Protein Resource (UniProt) in 2010. Nucleic Acids Res. 38, D142-D148 (2009).

59. Larkin, M. A. et al. Clustal W and Clustal X version 2.0. Bioinformatics 23, 2947-2948 (2007).

60. North, W. G., Fay, M. J. \& Du, J. MCF-7 breast cancer cells express normal form of all vasopressin receptors plus an abnormal V2R. Peptides 20, 837-842 (1999).

\section{Acknowledgments}

We are very grateful to Dr Brian Egleston for help with the statistical analysis in this study, and Ross Tomaino for help with the mass spectrometry analysis. We thank Heinrich Roder and Bill DeGrado for helpful suggestions regarding mapping of calmodulin-binding sites. This work was supported by Grants NIH R01 CA-63366 and R01 CA-113342; DOD W81XWH-07-1-0676 from the Army Materiel Command Tobacco Settlement funding from the State of Pennsylvania (to E.A.G.); NCI core Grant CA-06927; and support from the Pew Charitable Fund, to Fox Chase Cancer Center. Additional funds were provided by Fox Chase Cancer Center via institutional support of the Kidney Cancer Keystone Program.

\section{Author contributions}

O.V.P. performed the substantive majority of the experiments described, based on pilot studies performed by E.N.P. R.L.D. performed the structural prediction analysis. E.A.G. provided overall guidance on study design and execution, and wrote the paper.

\section{Additional information}

Competing financial interests: The authors declare no competing financial interests.

Reprints and permission information is available online at http://npg.nature.com/ reprintsandpermissions/

How to cite this article: Plotnikova, O. V. et al. Rapid calcium-dependent activation of Aurora-A kinase. Nat. Commun. 1:64 doi: 10.1038/ncomms1061 (2010).

License: This work is licensed under a Creative Commons Attribution-NonCommercialNoDerivative Works 3.0 Unported License. To view a copy of this license, visit http://creativecommons.org/licenses/by-nc-nd/3.0/ 